L'organisation de la lutte contre le sida au Cameroun : de la verticalité a la dispersion?

\title{
Fred Eboko
}

\section{OpenEdition}

\section{Journals}

Édition électronique

URL : http://journals.openedition.org/apad/184

DOI : $10.4000 /$ apad. 184

ISSN : 1950-6929

Éditeur

LIT Verlag

Édition imprimée

Date de publication : 1 juin 2001

Référence électronique

Fred Eboko, «L'organisation de la lutte contre le sida au Cameroun : de la verticalité a la dispersion ?», Bulletin de l'APAD [En ligne], 21 | 2001, mis en ligne le 28 février 2006, consulté le 07 septembre 2020. URL : http://journals.openedition.org/apad/184 ; DOI : https://doi.org/10.4000/apad.184

Ce document a été généré automatiquement le 7 septembre 2020

Bulletin de l'APAD 


\title{
L'organisation de la lutte contre le sida au Cameroun : de la verticalité a la dispersion?
}

\author{
Fred Eboko
}

1 Une des premières caractéristiques de la lutte institutionnelle contre le sida au Cameroun, comme dans beaucoup d'autres pays africains, était son caractère "vertical" (Gruénais, 1999 ; Kerouedan et Eboko, 1999). Les principes de l'action ont découlé peu ou prou, à partir de 1987, de la création et de la mise sous tutelle des Programmes nationaux de lutte contre le sida (PNLS) par le Global Programme on Aids (GPA) de l'Organisation mondiale de la santé (OMS). Au Cameroun, un autre facteur majeur a marqué l'élaboration de la politique publique de lutte contre le sida : l'instabilité de la direction du Programme, entre 1987 et 1998. En presque 15 ans de mobilisation étatique, le PNLS a connu des modifications récurrentes tant au niveau de sa direction que de la dynamique de son action. Un double changement de registre est intervenu dans la deuxième moitié des années 1990, au niveau mondial et au niveau local. La création de l'ONUSIDA en 1996, composé des principales agences des Nations Unies et de la Banque mondiale, devait prendre la relève du GPA pour conduire la coordination de la lutte contre le sida à l'échelon international.

2 Cette nouvelle armature répondait à la reconnaissance de la multisectorialité des incidences de la pandémie d'une part, et à l'échec relatif du GPA dans ses objectifs affichés au milieu des années 1980 d'autre part. Au Cameroun, c'est la nomination de l'ancien directeur régional de l'OMS pour l'Afrique, le Pro Gottlieb Monékosso, qui devait orienter de manière formelle l'organisation de la lutte contre le sida, dans un souci que l'on peut résumer en deux mots : coordination et décentralisation. Notre propos vise à mettre en évidence et à analyser les effets de la verticalité produite au niveau global sur la centralité des activités de lutte contre le sida au niveau local. L'effet de dispersion qui apparaît aujourd'hui dans le champ du sida au Cameroun doit être appréhendé comme une double conséquence de la conception internationale multisectorielle d'une part, et des difficultés vécues par l'organe central (le PNLS local) 
à coordonner des activités qui contrarient souvent ses velléités de décentralisation, d'autre part. En arrière plan de ces phénomènes, il faut aussi voir des habitus socio-historiques liés à une très forte centralité de Yaoundé, la capitale, vis-à-vis des provinces du pays.

3 L'intervention du ministre de la santé publique nommé en décembre 1997 consistait clairement à vouloir mettre un terme à l'instabilité du programme et à la personnalisation qui y a prévalu jusqu'à lors. Après 7 changements entre 1985 et 1998, le CNLS (Comité national de lutte contre le sida), qui est l'organe exécutif du Programme, est conduit par un comité collégial de 8 conseillers baptisé Groupe technique central du CNLS (GTC/CNLS), chargé d'assurer la coordination et la gestion du PNLS. Ce GTC est directement placé sous la responsabilité du Ministre de la santé publique, depuis sa création à la fin de l'année 1998. Le ministre devient de facto le Président du CNLS. La nouvelle ossature du CNLS ambitionnait de pallier les carences majeures que laissait constater l'historique de la lutte institutionnelle contre le sida au Cameroun. L'objectif portait, entre autres, sur la coordination et l'amélioration des activités relatives au sida, puis sur la décentralisation de la lutte en faveur des 10 provinces que compte le Cameroun. La tâche s'annonçait ardue dans un contexte de changement social et de crise économique. De ce fait, la nouvelle structure projetait de participer à la connexion de ses activités avec la réalité des dynamiques et des pratiques sociales sur lesquelles l'expansion de la pandémie se greffe depuis son apparition au Cameroun en 1985.

4 La diversité des rôles des conseillers, leurs compétences formelles puis leurs expériences respectives acquises sur le terrain semblaient manifester la prise en compte des écueils que le nouveau CNLS était appelé à combattre. Que ce soit l'intégration de l'expérience des personnes atteintes par le VIH/sida, la mise sur orbite de la formation permanente des acteurs, du souci de la pertinence des actions ou encore de la recherche fondamentale, le CNLS s'est fixé des orientations qui visaient à "localiser" la lutte sur l'ensemble du territoire et à harmoniser les rapports de l'univers du sida au Cameroun avec les partenaires internationaux. Un rappel sommaire de l'historique sera nécessaire pour éclairer les nouvelles perspectives. Ensuite, il sera utile de montrer à partir de notre enquête les incidences réelles de tous les facteurs évoqués précédemment au niveau des provinces, de telle sorte que l'on puisse mieux appréhender la tension et les contradictions entre la verticalité, la centralité et l'éclatement relatif du champ du sida dont la coordination relève d'un "grand écart permanent". Notre propos ne vise pas à aborder de manière exhaustive toutes les activités du PNLS mais il voudrait, à partir de certaines d'entre elles, dégager les contraintes internationales et locales qui pèsent sur le PNLS dans une période d'intense reformulation du débat international, que ce soit au niveau des Nations Unies ou des ONG.

1985-1997 : changement social, crise économique, instabilité du PNLS et progression exponentielle de l'infection a VIH

5 La fin des années quatre-vingts a coïncidé avec une crise inédite au Cameroun. Tous les indicateurs économiques entre 1985 et 1993 sont passés au rouge et le Cameroun a le triste privilège d'avoir été le pays africain à s'être le plus appauvri pendant cette période. Dans le même temps, le contexte politique a connu des remous intenses qui sont illustrés par une forte contestation du régime dès le début de la décennie quatre-vingt dix. C'est pendant cette crise économique majeure et la recherche d'un 
nouveau sens politique (démocratisation) qu'est née la lutte contre le sida au Cameroun. De 1985 à 1987 un premier comité voit le jour au ministère de la santé publique. En 1987, suivant les directives de l'OMS, le Cameroun crée le PNLS. Après une année de transition, s'ouvre le premier plan à moyen terme (PMTI) qui s'étale sur cinq ans. Après une année de transition (1993) s'ouvre le deuxième plan à moyen terme (PMT2) censé couvrir la période 1994-1998. Entre-temps, la direction du CNLS a changé 5 fois. Le taux de séroprévalence dans le même temps est passé de $0,5 \%$ en 1987 à $6,5 \%$ en 1998 dans la population générale. Aujourd'hui, en 2001, ce taux de séroprévalence au sein de la population dite "sexuellement active" est estimé à $11 \%$. Si la sécurité transfusionnelle a connu quelques résultats satisfaisants, la sensibilisation, puis la prise en charge (psychosociale et thérapeutique) des personnes vivant avec le VIH/sida a connu des fortunes diverses. La thématique catastrophiste assignée au sida, les difficultés de communication sociale envers le grand public et le déficit de coordination des activités liées à la lutte contre le sida ont témoigné des avatars du PNLS et de la relative anomie institutionnelle et politique qu'a connue la société camerounaise jusqu'en 1997. Les mouvements à la tête de l'Unité de Lutte contre le Sida (ULS), l'ancien organe exécutif du Comité, illustrent l'instabilité qui a prévalu à la tête de cette structure. La variable liée à la personnalité des autorités qui ont successivement dirigé le Programme explique que chaque départ correspondait aussi à bien des égards à une inflexion de la mobilisation, si ce n'est à un retour à la case départ. Le tableau résume le jeu "des chaises musicales" qui a rythmé la vie du CNLS.

Tableau 1 : Les directions des comités nationaux de lutte contre le sida

\begin{tabular}{|c|c|}
\hline Période & Direction du CNLS \\
\hline $\begin{array}{l}\text { 1985-1987 (1"' } \\
\text { Comité de suivi) }\end{array}$ & Acteur 1 Pr. Kaptué \\
\hline 1987-1993 & Acteur 1 Pr. Kaptué \\
\hline 1993-1994 & Acteur 2 Dr Salla Ntounga \\
\hline 1994-1995 & Acteur 3 Intérimaire Dr Mbouni Essomba \\
\hline 1995-1997 & Acteur 4 Dr Mpoudi Ngollé \\
\hline 1997 & Acteur 5 Dr Titsol (3 mois) \\
\hline $1997-1998$ & Acteur 6 Intérim assuré par la Direction de la santé communautaire \\
\hline $\begin{array}{l}1998 \\
\text { (Mars-Décembre) }\end{array}$ & Acteur 7 Pr. Ndumbe \\
\hline 1998 (Décembre) & $\begin{array}{l}\text { Acteur } 8 \text { Pr. Monékosso, ministre de la santé publique, président du CNLS } \\
\text { transformé en un Comité de } 4 \text { conseillers sous la responsabilité du } \\
\text { ministre. }\end{array}$ \\
\hline
\end{tabular}


Depuis 1999 (Février)

Acteur 8 président du CNLS , le ministre préside le CNLS composé du GTC (8 conseillers) et de 11 chargés de mission. Responsable du GTC: Mme le Pr. Shiro Koulla. Trois ministres se sont succédé entre-temps.

6 Jusqu'à sa nouvelle formule le Programme national de lutte contre le sida a donc été dirigé par sept présidents successifs. Chacun de ces médecins confirmés a tenté d'impulser à sa manière une dynamique, une politique avec des intensités et des capacités relationnelles très différentes. Après plus d'une décennie les résultats sont médiocres. Aucune catégorie sociale n'échappe à l'expansion de la pandémie. L'effectif des cas de sida se féminise et se rajeunit. Les zones rurales, d'abord relativement épargnées, subissent une montée significative des cas de sida. Le tableau 2 donne, à titre indicatif, la situation épidémiologique dans quelques villes du pays.

Tableau 2 : Prévalence du VIH/sida dans quelques villes

\begin{tabular}{|l|l|l|}
\hline Villes & Taux de séroprévalence & Population/Site sentinelle \\
\hline \hline Yaoundé (1) & $7 \%$ & Population générale \\
\hline \hline Bamenda (2) & $8-10 \%$ & Femmes enceintes \\
\hline \hline Limbe (2) & $10-12 \%$ & Femmes enceintes \\
\hline \hline Garoua (2) & $3-5 \%$ & Femmes enceintes \\
\hline \hline Bertoua (2) & $6-8 \%$ & Femmes enceintes \\
\hline
\end{tabular}

(1) Source : Projet de recherche multicentrique, IFORD/CHU/UCAC/CEPED, enquête été 1997.

(2) Source : Sam-Abbenyi Amos (supervised by), HIV/AIDS Surveillance Report 1996, Epidemiology Section, National Aids Control Program Cameroon, Ministry of Public Health, May 1997, 8p., multigr

7 Alors qu'au début de la reconnaissance de la pandémie de VIH/sida par les États africains le Cameroun semblait faire figure d'exception et être relativement préservé, la situation a bien changé aujourd'hui avec des taux estimés de prévalence générale qui ne cessent d'augmenter : 0,5\%, en $1988 ; 6,5 \%$, en $1998 ; 11 \%$, en 2000.

La centralité de l'état et la verticalité des directives internationales : vers un éclatement de la lutte contre le sida (1998-2001)?

8 Depuis décembre 1998, le Programme national de lutte contre le sida a pris de nouvelles orientations, sous l'autorité du ministre de la santé publique de l'époque, le Pr. Gottlieb Monékosso ${ }^{1}$. Dans un premier temps, il fonde un comité collégial de quatre conseillers, puis, en mars 1999, ce comité appelé Groupe technique central (GTC), est élargi à 8 conseillers qui supervisent et assistent 8 chargés de mission. Quelques dynamiques majeures marquent ce changement, notamment en ce qui concerne les personnalités nommées dans ce Groupe Technique Central.

L'ouverture et la volonté de crédibiliser l'action publique

9 La Présidente du GTC illustre, par ses compétences et par le symbole féminin qu'elle représente, la volonté d'imprimer un nouvel élan. Le Pr. Sinata Shiro Koulla devient 
ainsi Conseillère chargée du sida et du développement et dirige de fait le GTC. Elle est accompagnée par 7 autres conseillers dont la nomination est inédite dans le contexte institutionnel du sida au Cameroun. La première remarque qui s'impose est de souligner l'entrée dans cet organe exécutif de trois non médecins, choisis en fonction de la variété de leurs expériences respectives. Cette tendance semble induite par la volonté d'ouverture et de diversification du CNLS vers des acteurs sociaux susceptibles d'enrichir la problématique sociale de la lutte contre le sida. Dans le même temps, cette ouverture du CNLS, vise à obtenir des relais fiables au sein d'organes essentiels à la sensibilisation et à la communication. C'est le cas de la participation des personnes affectées et infectées par le $\mathrm{VIH} /$ sida, par le biais de la figure emblématique de ce groupe social (Conseiller au "Mode de vie ; Éthique et Droits de l'Homme") ; c'est aussi ce qui justifie l'incorporation d'un journaliste de renom au sein de cette structure (Conseiller à "l'Approche Pertinence"). Un acteur du PNUD assurera la "Relation avec les Partenaires". Ce qui témoigne de la stratégie d'ouverture du Comité vis-à-vis des bailleurs de fonds représentés à Yaoundé.

Néanmoins, si les sollicitations envers ces partenaires internationaux sont réelles, le nouveau CNLS semble avoir voulu se déconnecter du "face à face" entre lui et les bailleurs de fonds, de telle sorte que ceux-ci soient un soutien et non plus la condition sine qua non de toute initiative. En effet, même si cela paraît étonnant, il a fallu attendre la mise sur pied de ce GTC pour assister à des séances de travail, séminaires et autres rencontres publiques sur la seule initiative du CNLS. En d'autres termes, aucune réunion de ce type ne s'était tenue à Yaoundé avant cette date (et depuis au moins 1995) sans qu'elle ne soit l'œuvre ou l'initiative d'un partenaire étranger, public ou privé.

"La communication, la pertinence et l'éthique"

11 Parmi les impulsions récentes, deux nous semblent pouvoir être confrontées: la communication et l'éthique, réunies par un souci de "pertinence". Bien que ces thèmes soient affectés à deux conseillers différents, ils relèvent d'une volonté de réduire les carences accumulées depuis la création du Programme. La cooptation du président de l'association représentant les personnes atteintes est un événement exceptionnel. En effet, jusqu'à cette nomination ces personnes étaient tenues en marge des grandes réunions et, de fait, des décisions qui influençaient directement ou indirectement leur survie.

12 A partir des années 1998-1999, leur expérience en matière de counselling, leurs vécus, leurs itinéraires sociaux et thérapeutiques, puis leurs situations cliniques pourront servir à innover en la matière. Les droits essentiels de la majorité des patients sont régulièrement bafoués (transgression du secret médical, inégalités par rapport l'accès aux soins, exclusions sociales, etc.), et peu de gens se mobilisent pour les défendre.

Par ailleurs cette expérience, ces vécus doivent aussi servir à mieux sensibiliser et à démystifier le fait de vivre avec le VIH, dans un contexte où les représentations sociales assignent à la séropositivité une sentence de mort immédiate et une image nécessairement morbide. Par exemple, le fait que près de $10 \%$ des élèves d'un lycée d'une ville importante du Nord-Ouest estime qu'il faudrait "isoler" et/ou "éliminer" physiquement les séropositifs, donne une idée du travail qu'il reste à accomplir sur le terrain. Il paraît donc impérieux de sensibiliser au plus près (proximité des actions de sensibilisation, participation communautaire, etc.) tout en associant les personnes atteintes. Ceci doit être fait, non pas comme le voudrait le sens commun partagé par 
certains responsables sanitaires locaux (soit disant "pour montrer que le sida existe"), mais pour diffuser une double idée : prévenir et soutenir, sensibiliser la société et défendre ceux qui subissent l'infortune de vivre avec le VIH.

Pour induire ce type de changements, un souci de pertinence s'impose en matière de communication. L'entrée d'un journaliste, dont les compétences professionnelles et le charisme télévisuel sont reconnus, illustre cette volonté de rénovation et, surtout, le souci d'assainir une image institutionnelle que le grand public assimilait jusque-là à "la politique du ventre" et au "sida business". Pour autant, la volonté manifestée par ce journaliste d'une part, et l'énergie déployée par les personnes affectées et infectées par le VIH d'autre part ne vont pas sans tensions. Pour le représentant de ces derniers, les personnes atteintes doivent être au cœur du dispositif de la lutte. Pour le journaliste, on ne saurait passer du monopole des médecins qui a prévalu jusqu'ici, à un autre monopole : celui des porteurs du VIH. Sous cette dialectique se cache paradoxalement un espoir que les deux porteurs de ces projets vont sans doute réaliser bientôt, parce que leurs discussions illustrent une véritable nouveauté.

D'abord parce que leurs débats, comme ceux qui sont menés au sein du nouveau GTC, portent sur le "fond" et ensuite parce que leurs préoccupations convergent vers une prise de conscience collective accrue. Si les méthodes qu'ils préconisent semblent différentes, leur but est le même : démystifier la maladie et sensibiliser, défendre les droits humains et "démocratiser" la lutte contre le sida. En outre, l'image d'intégrité et de professionnalisme que le journaliste possède auprès du public, notamment auprès des jeunes, ne peut que participer à rompre avec l'image vénale assignée à la lutte contre le sida au Cameroun.

16 Cette nouvelle image est susceptible d'être fécondée par l'énergie combative reconnue dans l'univers du sida au représentant des personnes atteintes. Les deux collègues sont donc impliqués dans une action et une relation porteuses d'un changement potentiel. En matière de communication, la nouvelle équipe intègre de facto une réalité essentielle de la communication sociale : l'image du locuteur peut être aussi importante que le message qu'il édicte.

La dépersonnalisation : réalités, contraintes et impacts d'une volonté ministérielle

17 La volonté du ministre, nommé en 1997, de dépersonnaliser la direction du CNLS rejoignait le regard critique, scientifique ou profane, porté sur les multiples aléas subis par le Programme à la suite de tous les changements successifs de présidents. Le ministre Monékosso avait donc décidé d'être lui-même le président du CNLS en conférant le pouvoir exécutif du Programme au Groupe Technique Central. De cette manière, il semblait espérer qu'un changement éventuel à la direction du GTC n'aurait pas pour conséquence d'en réduire ou d'en anéantir les activités jusqu'à la nomination suivante. Un des effets attendus de cette volonté politique concernait la coordination des actions menées sur le sida au Cameroun. Cette coordination dont la responsabilité revenait au CNLS implosait littéralement à chaque changement de président dudit Comité.

18 Par conséquent, la logique ministérielle semblait frappée de bon sens, si l'on se référait à un fait : les changements de ministres de la santé publique n'ont pas été étrangers à la succession des présidents du CNLS. Il est inutile de rappeler que les remaniements ministériels ne dépendent pas des ministres mais des équilibres politiques et ethno-régionaux. De plus, à ce moment (1998-1999) on pouvait penser à bon droit que cette volonté de dépersonnalisation déjà en soi une marque de... personnalisation. En 
effet, il n'était pas excessif de penser que les avantages de cette dépersonnalisation dureraient sans doute le temps de l'action du Pro Monékosso à la tête de la santé publique. Au-delà de la volonté manifestée par ce dernier et au-delà de la pertinence des perspectives qu'il imprégnait à la politique de lutte contre le sida notamment, le phénomène de personnalisation est une constante de la vie politique en Afrique, du fait d'une institutionnalisation de l'État qui demeure plus un projet, un programme de travail, qu'une réalité tangible. Néanmoins, le Cameroun semblait être sorti de sa période de tumultes, de tensions politiques et d'instabilité administrative, à l'occasion du remaniement ministériel du 7 décembre 1997 (Eboko, 1999b). Celui-ci a clos une période de "transition politique" et le ministre de la santé, nommé à cette occasion, pouvait espérer que son action s'inscrirait dans la longue durée. C'est ce qui a le plus manqué à la lutte contre le sida au Cameroun depuis le début des années 1990. L'accalmie politique actuelle était donc susceptible d'aider la nouvelle impulsion ministérielle, pour que le sida devienne enfin un combat avec une cohérence et une stabilité administrative qui avaient déjà fait ses preuves ailleurs sur le continent (au Sénégal par exemple ; voir Delaunay, 1999).

Avec un recul de trois ans, il n'est pas trop tôt pour pouvoir dire que le ministre de l'époque avait eu une intuition politique intéressante. En effet, deux ministres lui ont déjà succédé en 2001 et ont été par voie de conséquence Présidents du CNLS. Aucun d'eux n'a tenu à changer la responsable du GTC qui dirige donc cet organe exécutif depuis 1998, avec pratiquement la même équipe. Le fait que la mise en œuvre du PNLS soit depuis cette date inféodée à la tutelle de chaque nouveau ministre dispense celui-ci d'une volonté de contrôle du champ (symbolique et matérielle) du sida qui se soldait à chaque fois par des changements à la tête du CNLS. Le "défi" de la constance au sein de la direction administrative du CNLS a donc été relevé grâce à la création du Groupe Technique Central qui est relativement vidé du contenu, des pressions, des nuisances ou des avantages politiques éventuels qui reviennent de facto au ministre. Celui-ci sait en outre pouvoir compter sur l'expérience et la connaissance du "milieu" acquises par le GTC qu'il chapeaute. Reste d'autres questions au sein du ministère.

La verticalité des décisions qui a présidé à la création du PNLS en 1987 s'illustre de manière manifeste dans l'organigramme du ministère de la santé publique. Alors que toute la structure du CNLS tente de baliser tous les pans nécessaires à l'action publique contre le sida, le ministère de la santé publique comporte, en marge du CNLS, des services compétents en la matière. Il en ainsi des bureaux "sida" et "MST" à l'intérieur du service du même nom ("Sida-MST"). Ce service est dirigé par une spécialiste reconnue pour ses compétences en matière de suivi et d'action préventive des MST et du sida, L'activité précédente de ce médecin au sein du bureau MST et sa pratique quotidienne au dispensaire des maladies vénériennes lui valent une réputation et une reconnaissance des acteurs locaux et étrangers travaillant sur la question du sida au Cameroun. En revanche, sa nomination à la tête du service "Sida-MST" la place en situation de "doublon" face au CNLS. Les deux bureaux mitoyens au sein de la direction de la santé communautaire du ministère de la santé publique à Yaoundé, semblent souffrir de problèmes de communication, dus eux-mêmes à une faible distinction des missions qui leur sont assignées. Ainsi, elle définit ses prérogatives comme étant celle d'un chef de mission devant conduire l'action contre le sida vers les délégations provinciales et les districts de santé, dans l'optique de la décentralisation qui est un des objectifs affichés par le CNLS. Pourtant, elle souligne que très peu de moyens lui sont affectés, très peu de missions lui sont confiées et que peu d'attentes sont manifestées 
par le CNLS à son égard. Contrairement à ses pairs, voisins et collègues du CNLS et en particulier du GTC, elle ne dispose ni de moyens de locomotion, ni de ligne téléphonique, donc d'aucun moyen efficace de communication. Elle "gère" un outil de travail quasi inexistant ${ }^{2}$. Sa position, les contraintes qu'elle subit et les ressentiments qu'elle exprime semblent en dire long sur le projet de décentralisation de la lutte contre le sida au Cameroun.

Le projet décentralisation

21 Parmi les grandes faiblesses de ]a lutte contre le sida, le déficit en matière de décentralisation est à souligner. Le CNLS prévoit donc formellement de réduire la verticalité et la centralisation des actions. Pour cela les provinces, puis les districts de santé sont appelés à devenir des maillons de base de la lutte décentralisée et non plus les derniers servis. La décentralisation budgétaire est une question épineuse que le GTC vise à résoudre. C'est un défi important au vu des enjeux que la ventilation des moyens financiers peut générer dans un contexte de pénurie. Ainsi, la lutte contre le sida devra s'appuyer sur des EPLS (Équipes provinciales de lutte contre le sida) et sur des EDLS (Équipes de districts de lutte contre le sida). La réalité paraît imposer des contraintes socio-historiques locales et internationales plus lourdes. Notre enquête de terrain dans quelques provinces camerounaises illustre les difficultés auxquelles se heurtent les velléités de décentralisation.

La question de la décentralisation occupe les débats et les textes administratifs au Cameroun, depuis les indépendances. Si la volonté affichée a toujours existé, elle s'est toujours heurtée à un autre mouvement: la volonté de contrôle de la part des ministères et la très forte centralité de l'État au Cameroun. Cette dynamique historique est elle-même liée au fait que le régime d'Ahidjo (1958-1982) s'est construite sur une volonté de centralité dans ce pays qui était une république fédérale (depuis 1961) devenue une "république unitaire" en 1972 en réduisant au maximum toutes les velléités d'autonomie, même relatives, au profit du volet "unitaire" qui a toujours contrarié la nécessité de décentraliser l'action étatique. De la même manière, les autorités qui dirigent les "délégations provinciales" sont elles aussi mues par des "habitus" de centralité. C'est-à-dire qu'elles exigent souvent pour conduire ou avaliser des projets qui leur sont soumis au niveau local, l'autorisation ou l'accord des autorités de Yaoundé dont les délégués provinciaux se plaignent par ailleurs de ne pas assez "décentraliser". Sur le papier, le maître mot est toujours la "décentralisation" qui se décline sur le terrain d'une manière très variable, entre "délégation", "déconcentration" et "dévolution" 3 . Il convient de s'attarder sur les tensions et les contradictions empiriques et avérées qui se manifestent entre les volontés officielles et les dynamiques concrètes, en matière de "division sociale du travail", dans la lutte contre le sida.

La décentralisation de la lutte contre le sida au Cameroun : entre délégation, déconcentration, dévolution et "abandon"

Les chartes, les directives et les conventions internationales concernant la santé ont été successivement ratifiées par le Cameroun. Les Conférences d'Alma Ata (1978), de Lusaka (1985), d'Harrar (1987) et de Bamako (1987) ont toutes été suivies de conventions signées par le gouvernement camerounais. Une grande part de ces directives internationales prônait un processus de décentralisation. Il en de même pour l'adhésion formelle aux directives issues des conférences internationales sur le sida les MST en Afrique. Pour autant, ces décisions internationales qui suivent la logique de la 
"verticalité" se heurtent, depuis toujours, à la résistance administrative et à la très grande force centrifuge de l'Etat camerounais.

Dans ce cadre, la stratégie de lutte contre le sida inscrit le district de santé comme cellule de base du système de santé. A la décentralisation géographique de l'administration camerounaise qui correspond à une "déconcentration" (la forme la moins poussée de la décentralisation), s'est joint une décentralisation fonctionnelle, en ce sens que chaque délégation provinciale est supposée assurer les prérogatives administratives que le ministère représente au niveau national. Dans les faits, qu'il s'agisse de santé ou d'autres domaines ministériels, la "décentralisation" représente plus un "projet" qu'une réalité au Cameroun.

Une appréhension empirique, même rapide, permet de constater que tous les stades et tous les types de "décentralisation" sont représentés sur l'échiquier de la lutte contre le sida au Cameroun, au gré des interventions étrangères, multilatérales, bilatérales et des organismes privés. Ainsi, nous assistons à des schémas qui mettent en lumière des chevauchements entre "décentralisation", "déconcentration", "dévolution" et "privatisation". Chacune de ces figures est présente dans la répartition structurelle des actions de lutte contre le sida, par les effets des différentes interventions qui font du PNLS un acteur parmi d'autres ; pour une politique publique qui se fait à la "marge" par des partenaires étrangers, même si elle est supposée être édictée par le "centre". Notre terrain permet d'illustrer ce phénomène d'atomisation structurelle qui rend difficile la coordination qui est une des fonctions principales et officielles du PNLS.

Actions sanitaires et dynamiques épidémiologiques : le syndrome de la dissonance dans la lutte contre le sida.Centre et périphérie d'une dynamique internationale : des inégalités géographiques

L'action contre le sida au Cameroun est corrélée à la présence et à la dynamique des organismes internationaux, des agences de coopération bilatérale et multilatérale et, enfin des ONG internationales (Eboko, 1999a). La GTZ couvre trois provinces: le Littoral, le Sud-Ouest et le Centre. Elle y finance les projets d'IEC et surtout soutient les différentes activités de l'association des séropositifs et leurs proches dont elle est le principal support financier. La Coopération française vient de financer la création d'un hôpital de jour à Yaoundé destiné aux patients atteints du VIH/sida. Le Fonds d'aide et de coopération (FAC, France) couvre la province du Nord pour les activités d'IEC et les initiatives en matière de prise en charge des patients de cette province, avec la participation de la principale association de personnes vivant avec le VIH, l'AFSU (Association des frères et sœurs unis) de Yaoundé dont les membres sont en train de créer une antenne dans le chef-lieu du Nord, Garoua. Dans ces différents cas, il s'agit d'un transfert de facto d'une compétence sectorielle (santé et sida) du "centre directeur de la société" (l'État, suivant la formule durkheimienne) vers des agences de coopération. C'est une logique que l'on peut qualifier de "décentralisation par défaut" ou encore de "dévolution conjoncturelle". En effet, c'est la conjoncture économique et administrative d'un État rétracté en son centre qui permet aux organismes étrangers de s'installer où ils le souhaitent et d'initier des actions relativement autonomes vis-à-vis de celles conçues par le PNLS à Yaoundé.

Les autres provinces sont sujettes à un "vide" relatif que tentent de combler des actions de missions chrétiennes, comme à Kumbo, petite localité du Nord-Ouest, où l'hôpital de la Mission catholique héberge des personnes infectées par le VIH ou atteintes du sida, dans une logique que nous pourrions qualifier de "privatisation partielle". Les 
frontières camerounaises sont particulièrement pénalisées par leur éloignement $\mathrm{du}$ "centre" du pays et, de fait, du "Cameroun utile". Ici, nous pouvons parler "d'abandon". L'ONUSIDA, le CNLS et les acteurs locaux : une coordination à plusieurs vitesses, des collaborations ambivalentes

L'entrée en lice de l'ONUSIDA au Cameroun, en tant que représentation permanente sur le territoire camerounais, date de la fin 1999-début 2000. Cette mission a été assignée au Dr Emmanuel Gnaoré, ancien directeur du CNLS ivoirien et ancien responsable ONUSIDA à Madagascar. D'emblée le représentant ONU SIDA à Yaoundé souligne la principale difficulté rencontrée face au CNLS et aux acteurs camerounais :

"Ce pays a des ressources humaines et des compétences énormes mais il est très difficile de faire travailler les Camerounais ensemble. C'est incroyable de constater que nous essayons de mettre en commun les acteurs camerounais compétents dans le domaine du sida autour de la responsable du OTC et que ce sont les Camerounais qui exigent que nous (ONUSIDA) soyons là pour coordonner ces rencontres (...). Tout se passe comme si les uns et les autres avaient besoin d'un étranger pour éviter que ce soit l'un d'eux qui le fassent. C'est étonnant (... )" 4 .

Partant de ce constat, l'ONUSIDA a pris, en quelque sorte, les rênes de l'initiative en matière de mobilisation sociale contre le sida. Le premier effet tangible de cette prise en main est symptomatique des difficultés du PNLS. Ce que celui-ci n'a pas réussi en 15 ans, l'ONUSIDA l'a réalisé en quelques mois: mobiliser de manière permanente la presse locale. Depuis le début de l'an 2000, les principaux journaux de Yaoundé affichent à leur "Une" un message de sensibilisation contre le sida. Nous avons croisé nos sources et entretiens entre un de ces journaux (Mutations) et l'ONUSIDA pour mieux comprendre le phénomène. Deux critères ont prévalu pour arriver à cette mobilisation. Le premier est d'ordre financier; l'ONUSIDA finance ces messages au titre d'encarts publicitaires.

Le second critère qui explique pourquoi cette mobilisation n'a pu être induite par le PNLS relève de l'appréhension politique de la question du sida au Cameroun. Le PNLS est assimilé à un récepteur de la "manne" qui serait générée par les bailleurs de fonds internationaux. Cette idée que le PNLS et ses dirigeants successifs bénéficient de ressources très importantes est une idée ancrée dans les représentations sociales de la lutte institutionnelle contre le sida, assimilée à un maillon de réseaux internationaux où circulent des sommes que l'ensemble des acteurs sociaux jugent ou estiment "considérables". Même si les fonds attribués au PNLS ont baissé depuis 1995 (ce qui s'est aussi traduit par une "vacance" à la tête du PNLS entre 1996 et 1998), l'idée de la "richesse" du PNLS est restée identique.

De fait, pour les journalistes toute action en faveur de la lutte contre le sida et initiée par le Comité devait être assortie d'un financement conséquent. Pour sa part, le PNLS avait déjà réuni, en 1995, les principaux acteurs de la presse nationale (écrite, radiophonique et télévisuelle) pour les sensibiliser à l'action commune contre l'expansion de la pandémie au Cameroun. La réunion s'était alors terminée par des demandes de financement émises par chaque média que le PNLS était censé présenter à des bailleurs de fonds. Cette réunion s'était finalement soldée par une inertie générale des médias en attente de soutien financier, y compris dans des activités qui relevaient du travail classique de journalistes (reportages, informations générales, etc.) (Eboko, 1999a, 2000). Pour autant, nous ne saurions conclure de ces faits que la prise de conscience journalistique date de la présence permanente de l'ONUSIDA. En effet, les mêmes journaux qui font payer à l'organisation internationale des encarts publicitaires 
sur le sida, font passer des articles d'analyse de la situation, des interviews d'acteurs, des reportages et des informations ponctuelles. La raison de cette "schizophrénie" apparente relève de la distinction que font les journalistes et les autres acteurs entre les personnalités non institutionnelles (supposées être en dehors du champ d'éventuels profits) et celles qui émargent au PNLS ou auprès d'organismes étrangers directement et statutairement liés à la lutte contre le sida.

Dans cette logique, l'ONUSIDA enregistre un crédit lié à sa visibilité et à la dynamique impulsée par le Dr Gnaoré. A l'inverse, il devient de plus en plus difficile de comprendre ce que coordonne le CNLS, si ce n'est d'accompagner les initiatives de l'ONUSIDA. Ainsi une nouvelle signature a vu le jour sur tous les projets initiés à Yaoundé, UNAIDS-CNLS, assortie à chaque fois de l'agence particulière de l'ONUSIDA à l'origine d'une action particulière: UNESCO, UNICEF, PNUD, OMS ou Banque mondiale. La succession de réunions de coordination ou de sensibilisation organisées depuis le début de l'année 2000 ont succédé à celles du GTC en 1999.

La lutte connaît donc une nouvelle dynamique qui met en évidence un effet pervers fondamental: la centralisation des actions et des acteurs à Yaoundé et la relative absence de projets dans les provinces qui ne sont pas couvertes par un partenaire bilatéral comme la Coopération française (Centre et Nord du pays) ou l'agence de coopération technique allemande (Centre, Littoral, Nord-Ouest et Sud-Ouest).

Nous pouvons donc en conclure que la centralisation de l'action sur le sida à Yaoundé est le corollaire de la verticalité des directives et des initiatives internationales. Quelques exemples issus de notre travail sur le terrain peuvent illustrer cette analyse pour laquelle nous allons utiliser le cas des provinces du Sud-Ouest et du Nord-Ouest (les deux provinces anglophones).

Limbé et Bamenda face au sida : marginalisation et instrumentalisation

En 1996, des enquêtes sentinelles auprès de femmes enceintes à Limbé et Bamenda indiquaient que ces deux villes étaient les plus atteintes par le VIH/sida (Sam Abenyi, 1997). A cette époque, les estimations officielles chiffraient le taux de séroprévalence auprès des 15-49 ans dans une fourchette entre 10 et $12 \%$ à Limbé, et entre 8 et $10 \%$ pour Bamenda (aujourd'hui les chiffres indiquent des taux supérieurs à $14 \%$ pour les deux villes). Nous nous sommes rendus dans l'une et l'autre de ces villes où nous avons successivement interrogé les responsables de la santé et enquêté auprès d'une de leurs cibles privilégiées, les scolaires. Il était question d'appréhender la politique publique de lutte contre le sida à l'échelon provincial et à celui du district et de comprendre les dynamiques sociales et les représentations vis-à-vis de la maladie chez les jeunes. Les questions du district de santé et celle de la décentralisation vers cette entité ont été un arrière plan important qui permettait de saisir ces problèmes dans leur globalité.

Limbé : la cité sinistrée, la double "victime" d'une décentralisation manquée

Les chiffres disponibles au moment de notre mission à Limbé (1999) sont ceux que nous avons annoncés plus haut : 10 à $12 \%$ de séroprévalence du VIH/sida à Limbé. C'est donc par ces chiffres que nous avons justifié notre intérêt pour le district de santé de Limbé en introduction à notre entretien avec le médecin chef de ce district de santé. Nous voulions savoir, pour commencer, comment elle expliquait le choc épidémiologique subi par Limbé et les réponses envisagées sous sa responsabilité. L'exploitation de cet entretien et notre analyse vont de la géographie aux contradictions administratives et symbolisent toute l'ambivalence de la politique publique qui nous occupe ici. 
D'un point de vue géographique et démographique, Limbé possède un pouvoir d'attraction important puisqu'il s'agit d'une station balnéaire située au bord de l'océan Atlantique et au pied du volcan le plus célèbre du pays, le Mont Cameroun (4070 m). A cela il faut ajouter la présence de deux grandes sociétés dans la région de Limbé ; la Cameroon Development Corporation (CDC) et la Société nationale de Raffinerie (SONARA).La responsable du district de santé estime, à juste titre que le tourisme attirait une forte circulation démographique sur la commune. Elle y a ajouté que la prostitution était un facteur important de la circulation du virus. Pour ce qui concerne les réponses sanitaires et politiques apportées à la crise épidémiologique de Limbé, le médecin chef de district a souligné le peu de moyens matériels dont elle disposait. Au-delà de l'entretien, Limbé est un cas d'école qui explique et illustre les avatars d'une décentralisation ambiguë. D'une part, c'est la ville la plus peuplée de toute la province du Sud-Ouest dont elle est aussi la préfecture, et d'autre part, cette station balnéaire n'est pas le chef-lieu de province qui est à Buéa, une petite ville située à une demi-heure de route de Limbé.

39 La première conséquence de cette distinction qui date de l'époque de la colonisation allemande (Buéa fut la capitale du Kamerun allemand) est que Limbé n'est que la seconde ville de la province d'un point de vue administratif. De fait les moyens alloués à chaque province passe d'abord par le chef-lieu où se situe la délégation provinciale de la santé publique (comme toutes les délégations ministérielles) avant d'être affectés aux districts sanitaires. Deux tutelles administratives altèrent donc les moyens attribués au district de Limbé ; Yaoundé (la capitale politique du pays) et Buéa (le chef-lieu de la province).

Ensuite, le partenaire étranger qui œuvre dans cette province, au titre de la coopération germano-camerounaise (la GTZ) situe ses actions en priorité dans "la capitale provinciale" (Buéa) conformément aux accords passés entre les deux pays. De fait, les actions menées contre la tuberculose et le sida dans la province sont concentrées à Buéa et dans les localités avoisinantes. Limbé se retrouve avec le "record national" de séroprévalence et des moyens modiques pour faire face à la pandémie. Le caractère urgent de la situation épidémiologique de Limbé ne semble pas changer les stratégies d'attribution des moyens que ce soit de la part de Yaoundé, de Buéa ou de la coopération internationale. Le CNLS prônait l'institutionnalisation des EDLS (Équipe de district de lutte contre le sida); à Limbé, l'équipe est en place, comme le souligne la responsable qui montre dans le même temps que cette mise en place ne résous pas le problème de la mobilisation sanitaire. Pour montrer à quel point la situation de son district est éloignée des autorités qui dirigent le PNLS. la responsable du district raconte un fait qui parle de lui-même. En 1998, alors que la situation dramatique du district est connue, le CNLS organise un séminaire de formation et de sensibilisation à Limbé. Ce qui aurait pu paraitre comme un choix symbolique judicieux s'est avéré être un hasard symptomatique et malheureux puisque la responsable du district n'a pas reçu d'invitation à participer à cette réunion. Le lieu avait été choisi manifestement pour des raisons touristiques et les organisateurs avaient "oublié" successivement la situation sanitaire de la ville d'accueil et d'inviter sa responsable.

41 Peut-on parler du sida au Cameroun, en se réunissant à Limbé, et oublier que c'est la ville la plus éprouvée par la maladie? Peut-on organiser un séminaire sur le sida au Cameroun sans penser à Limbé tout en étant à Limbé ? Limbé site touristique peut-elle faire oublier Limbé la sinistrée à des responsables de la lutte contre le sida? 

Pour clore ce tour d'horizon, il n'est pas seulement anecdotique de signaler que le district de santé de Limbé avait un budget de 60.000 FCFA pour organiser la Journée mondiale de lutte contre le sida, pour l'année qui a précédé notre enquête. Dans ce budget, la moitié était un don d'une association locale. La modicité de ces sommes est assez explicite pour signifier la dissonance entre les velléités de décentralisation et les réalités sanitaires relatives au sida au Cameroun. A l'inverse, le fait que la responsable exige une autorisation formelle de Yaoundé pour conduire une enquête ou un projet sur son district (contrairement aux prérogatives attribuées officiellement à chaque circonscription sanitaire) indique la complexité d'une situation où se mêlent la marginalisation et un besoin de reconnaissance par le centre. Cela pourrait altérer une véritable dynamique de décentralisation, en renforçant l'inféodation à la tutelle du centre dont elle souligne par ailleurs la nuisance. De ce point de vue, Limbé est le symbole des carences du système et un cas d'école en matière de succession de contradictions administratives, historiques et sanitaires.

Bamenda : déconcentration, instrumentalisation

Notre investigation en zone anglophone s'est achevée à Bamenda, la capitale provinciale du Nord-Ouest. Les logiques de la lutte contre le sida à Bamenda mêlent des dynamiques associatives, sanitaires, administratives et des enjeux Nord-Sud. L'imbrication des dimensions locales et internationales a conduit une de ces associations à devenir le principal opérateur privé de la lutte contre le sida dans la province du Nord-Ouest, y compris auprès des scolaires: le FAS Foundation (Fight Against Aids and STD's). C'est cette association qui contrôle les 58 "Clubs Santé" de la province du Nord-Ouest, à la suite de protocoles passés avec les délégations provinciales de la santé et de l'éducation, puis avec l'UNICEF et l'OMS. Outre le fait de distribuer du matériel à ces "Clubs Santé", le FAS y organise des séminaires de formation des responsables scolaires de ces clubs, placés sous la tutelle d'un enseignant, en général d'un professeur de biologie. Le FAS met en outre à la disposition des élèves l'accès Internet dont il dispose ${ }^{5}$.

Les activités du FAS Foundation à Bamenda concernent l'accès de patients, sans frais de leur part, à des polythérapies. Dans un pays frappé par la crise économique et la paupérisation de la majorité de sa population, cette action n'a pas manqué de nous interpeller lors de notre enquête. La mise sous bithérapie des patients en question illustre des enjeux qui concernent plusieurs personnes et plusieurs instances nationales et étrangères. En effet, les patients mis sous bithérapies participeraient à une recherche vaccinale menée pour le compte de l'Université du Missouri, qui avait signé un accord avec le PNLS. De fait, les patients sont soumis à un "Vaccine Project" impliquant un accord entre la Faculté de Médecine de Yaoundé, l'Université du Missouri, le FAS Foundation qui reçoit et assure le suivi des malades. C'est ce suivi qui serait susceptible de poser problème. En effet, l'épidémiologiste qui nous a reçu précise qu'il prescrit lui-même les traitements, suite à une "formation clinique" qu'il a reçue. Un médecin passerait une fois par mois à l'association pour les consultations. Plusieurs questions se posent ici et concernent : l'association des molécules (deux antiprotéases, saquinavir + nelfinavir) et la compliance des patients pour un traitement où celle-ci est capitale. Il conviendrait de suivre l'évolution de ce protocole qui met en jeu de manière fondamentale l'introduction des antirétroviraux au Cameroun. 
Au-delà des questions médicales et du "colloque singulier" entre patients et praticiens, le projet dont il est question ici soulève le problème des entreprises sanitaires qui parcourent le champ du sida au Cameroun. Ces projets et initiatives rendent presque caduque la question de la décentralisation, en ce sens que cette dernière est absente des préoccupations et des projets des "entrepreneurs sanitaires". Ces initiatives permettent à des "aînés sociaux" (par exemple, des professeurs de médecine) de se positionner dans des réseaux transnationaux. La "filière" constituée par l'Université du Missouri à Kansas City, la Faculté de médecine de Yaoundé et l'association FAS Foundation à Bamenda n'intègre que très peu la délégation provinciale de la santé publique du Nord-Ouest sise à Bamenda. Au vu du document qui nous a été soumis (l'accord de partenariat), cette dernière n'est partie prenante en aucune manière. Le site se prêtait au projet (plus de $14 \%$ des adultes sont estimés séropositifs à Bamenda) et l'expérience de terrain de l'association FAS représentait une ressource dynamique et pratique pour le recrutement des patients. Le fait que le délégué provincial de la santé soit très actif auprès des associations ne change rien à la question puisqu'il s'agit ici d'une activité où se mêlent fonds privés et publics pour des enjeux de santé publique et privée à travers lesquels l'instance exécutive de la province observe davantage qu'elle ne décide.

Les associations montrent à leur tour des processus de capitalisation des partenaires. L'exemple des associations est à ce titre significatif et illustre une régulation d'abord verticale de la lutte contre le sida et ensuite une répartition géographique inégale qui s'effectue au gré des desiderata de ces structures internationales. Ces dernières répondent à des objectifs qui ne sont pas directement motivés par des réalités épidémiologiques du pays.

66 associations répertoriées par le PNLS, les deux tiers (51) sont basés à Yaoundé, la capitale administrative et politique, et dans la province du Centre qui en compte au total 53. Au-delà du listing officiel, d'autres structures associatives et actives ne figurent pas dans la liste du PNLS.

Sur les dix provinces administratives que compte le pays, quatre ne comportent officiellement aucune structure associative relative au sida: l'extrême Nord (à la frontière $\mathrm{du}$ lac Tchad), la province du Nord, l'Est, province frontalière de la République centrafricaine, et le Sud, qui est représenté sur la liste par un cabinet médical qu'on peut difficilement considérer comme une association. Douala et la province du Littoral comptent quatre associations. Limbé et le Sud-Ouest possèdent quatre organisations de ce type. L'Ouest et Bafoussam, son chef-lieu, sont représentés par une association dont le président n'est autre que le vice-président d'une ONG internationale basée à Yaoundé. Cette ONG internationale est répertoriée à Yaoundé et on peut penser que l'association de Bafoussam, vu ses objectifs affichés, est un continuum provincial de l'organisation sise dans la capitale, même si les dénominations officielles diffèrent. Le Nord-Ouest et Bamenda possèdent deux ONG. Une enquête menée sur place a permis de rencontrer trois autres structures très actives, non recensées par les services de Yaoundé. Enfin, la province de l'Adamaoua (voisine du Centre et du Nord) compte une association de développement rural ayant choisi de mener des campagnes d'IEC.

Un document récent du bureau de l'UNESCO à Yaoundé confirme la tendance de la centralisation des associations à Yaoundé. Sur les 34 associations pour la jeunesse qui ont demandé l'aide de l'Unesco en 2001, 28 sont situées à Yaoundé ${ }^{6}$. Cette répartition géographique suit une logique duale entre la proximité des organismes internationaux 
et les initiatives de mobilisation contre le sida. C'est l'illustration du projet sida conduit par l'Église évangélique du Cameroun (EEC) et l'ONG française "L'Appel".

Le programme sida EEC-L'Appel: une illustration de l'enjeu Nord-Sud. En 1994, le "département jeunesse" de l'Église évangélique du Cameroun (EEC) à Douala et l'association française "L'Appel" lancent un programme sida. L'Appel finance des campagnes d'IEC et la formation des jeunes animateurs issus de la jeunesse évangélique de Douala. Les financements de L'Appel pour ce programme sont assurés par l'Union européenne. Après cinq années d'activités, le programme comptait 200 animateurs opérationnels dont les actions se répartissent sur 12 régions synodales et 30 antennes correspondant chacune à un arrondissement camerounais. L'action circonscrit deux grandes provinces camerounaises, le Littoral et l'Ouest. Les jeunes de ce programme sida sont de fait inféodés à deux tutelles : celle de la hiérarchie de l'Église et celle des partenaires parisiens. Ce double ancrage place les animateurs bénévoles dans une position que les conflits au sein de la hiérarchie de l'EEC rendent inconfortable. Leur formateur et interlocuteur au sein de l'église, un médecin, subit la tutelle de l'autorité proprement religieuse qui voudrait avoir un contrôle plus direct sur le programme sida et les jeunes. Dans le même temps, les partenaires parisiens tentent de passer outre ces conflits en insistant sur l'action des jeunes qui doivent rendre des comptes, conformément au contrat entre l'EEC et L'Appel. Les déplacements de ces jeunes (qui se répartissent en étudiants et jeunes travailleurs) et leurs activités personnelles représentent, pour les acteurs français, des soucis qui semblent majeurs. L'action sida intervient ici dans un contexte où ces jeunes (de 18 à 30 ans) essaient de s'autonomiser par des voies multiples, avec les contraintes sociales récurrentes dans les villes africaines. L'action contre le sida relève de choix spirituels mais est aussi le moyen d'être en contact avec "l'étranger" par l'intermédiaire des deux médecins français qui sont leurs principaux interlocuteurs réguliers.

51 L'impression qu'ont ces jeunes d'être "surveillés" plus que de raison n'est pas fortuite. Ils sont au centre d'enjeux dont ils sont simultanément un maillon lointain et la ressource humaine principale. La diversification des activités que L'Appel souhaite apporter au programme (prise en charge psychosociale des personnes atteintes) les place en position de force grâce au partenaire français mais les met dans une posture délicate face aux désaccords des "anciens" de l'église. En définitive, le programme sida de l'EEC-L'Appel représente donc l'interface de trois logiques qui peuvent conduire à une aporie: L'Appel finance ce programme sida grâce à l'apport de la coopération française et de l'Union européenne (100 millions de francs CFA en cinq ans), les cadres de l'EEC fournissent et encadrent les jeunes bénévoles qui agissent sur le "terrain" au nom de leur foi religieuse et des liens "fraternels" renforcés par cette mobilisation commune. À la fin de l'année 1999, le projet arrivait officiellement à son terme pendant que chacune des trois parties en question se demandait si l'action entreprise contre le sida survivra à l'arrêt des financements.

En effet, le leader de l'association (âgé de 25 ans en 2001) a quitté le Cameroun durant l'été 1999, ne voyant pas, suivant ses dires, la suite qui allait être donnée au Programme. Après un passage en France pour une rencontre ecclésiastique dans le sud du pays, il s'est soustrait du groupe avant le retour au Cameroun et a rejoint un autre Etat de l'Union européenne. A Douala, le programme n'a pu démarrer, les autorités de l'EEC n'ayant pas, faute de moyens, continué l'action entreprise sous l'égide de l'Appel. Entre-temps, le jeune homme qui a coordonné toute l'action des jeunes pendant cinq 
ans, a réussi à obtenir un permis de séjour en 2000 dans le pays où il réside. Il y a trouvé du travail et exerce le métier pour lequel il avait obtenu un CAP à Douala. Il est entré en contact avec des organismes de lutte contre le sida grâce auxquels il envoie quelques plis à son association: brochures, préservatifs, etc. De son engagement aux côtés des anciens de l'église de Douala et des médecins de l'Appel, il garde un souvenir mitigé, même si l'expérience personnelle lui a été "très bénéfique". Il souligne: "chacun a essayé jusqu'au bout de protéger ses intérêts..." Il constate que son "avenir personnel" n'était la préoccupation de personne, sinon les partenaires parisiens l'auraient aidé à suivre une formation en France. Au lieu de quoi, précise-t-il, ces derniers "voulaient [le] fixer au Cameroun parce que c'était dans leur intérêt... ". Son intérêt, dit-il encore était de sortir d'une situation où le projet qu'il animait à temps plein lui rapportait 25.000 FCFA par mois pour aller vers une activité qui lui permette réellement de subvenir aux besoins de ses huit frères et sœurs dont il est l'aîné. Son expérience est de celles qui justifient qu'un des nouveaux enjeux de la lutte contre le sida consiste à intégrer celle-ci dans des plans plus globaux de lutte contre la pauvreté.

Pour conclure : la lutte contre le sida au Cameroun, un modèle dissonant de politique publique

53 L'aggravation de la situation épidémiologique du sida au Cameroun et l'échec de la prévention ont induit des modifications formelles au niveau des organisations internationales. A la faveur de l'action de la Banque mondiale auprès de l'ONUSIDA d'une part, et d'autre part des tractations du gouvernement camerounais pour la révision de la dette du pays auprès des institutions dites de Bretton Woods; un accord de principe a été établi en 2000. Le Cameroun se devait de soumettre un plan de lutte contre la pauvreté à la Banque mondiale et, dans le même élan, de soumettre à l'ONUSIDA un plan stratégique d'urgence de lutte contre le sida. Le premier aspect de cette négociation internationale devait concourir à l'admission du Cameroun au sein du groupe des Pays pauvres très endettés (PPTE), ce qui permettrait l'annulation de sa dette. Le second aspect de ces engagements diplomatiques devait permettre à la Banque mondiale d'attribuer des fonds importants au Cameroun pour la lutte contre le sida. L'année 2000 a donc été riche en agitations internationales au Cameroun, avec une excitation supplémentaire liée à l'imminence du Sommet des chefs d'État de France et d'Afrique à Yaoundé (janvier 2001). La rencontre entre le gouvernement camerounais, la Banque mondiale et l'ONUSIDA eut lieu le 12 septembre 2000 au Palais des Congrès de Yaoundé. Le Cameroun, par l'entregent du travail du CNLS, a donc présenté son "Plan stratégique d'urgence contre le sida". Il s'est agi en fait d'une déclaration d'intention qui suffisait à la Banque mondiale pour libérer des fonds. La lecture de ce document montre qu'il ne comporte aucune stratégie nouvelle par rapport à tous les plans précédents. Les groupes cibles sont restés les mêmes depuis le début des années 1990 : les "jeunes", les prostituées, les transporteurs, etc. (Eboko, 1996). La méthode choisie n'a pas varié non plus, elle s'est dotée d'un nouveau slogan : "100 \% condoms". Au-delà du sida, cette rencontre et les procédés en question sont conformes aux négociations entre les États africains et les bailleurs de fonds internationaux, en ce qui concerne les demandes de financements ou de révision de la dette. Un fonctionnaire de la Banque mondiale illustre ces face-à-face diplomatiques par une phrase riche de sens que l'on pourrait appliquer à la rencontre de Yaoundé : "Nous faisions semblant de croire ce qu'ils faisaient sembler de penser". d'urgence" ne signifie pas qu'il n'y a pas eu d'évolution dans la lutte contre le sida au 
Cameroun mais que le "combat" se passe assez largement ailleurs, notamment à travers les actions des différents partenaires internationaux. Ceux-ci se fixent chacun leurs objectifs, lesquels finissent par orienter, nourrir et impulser la politique publique de lutte contre le sida. Entre la verticalité des directives internationales, l'atomisation des projets et la centralisation des actions sur Yaoundé et quelques régions choisies par les partenaires étrangers, le CNLS se retrouve à. assumer un rôle de coordination que tous ses partenaires contrarient. D'où la place importante qu'occupe l'ONUSIDA et ses représentants à Yaoundé, puisque ce sont eux qui orientent, stimulent et coordonnent officieusement cette politique publique dont l'action officielle revient au CNLS et, en particulier au Groupe Technique Central. Par exemple, la question des polythérapies antirétrovirales a donné lieu à la constitution officielle d'une plate-forme de grossistes pharmaceutiques qui sont les seuls à pouvoir vendre ces molécules, pour éviter une inflation dans ce domaine. Or, il se trouve que plusieurs projets mettent des patients sous polythérapies à des prix modiques ou gratuitement, suivant des motivations diverses. L'ONG Médecins Sans Frontières Suisse a lancé un programme de mise sous tri thérapies qui devrait à terme concerner trois cents patients à Yaoundé (Projet baptisé "Parvy"). Le FAS de Bamenda s'occupe d'une centaine de patients sous bithérapies. Un projet similaire au projet Parvy est conçu pour Douala avec la participation du laboratoire pharmaceutique MSD (Projet "Darvi"). A ces dynamiques on peut rajouter l'investissement des grandes sociétés parapubliques ou privatisées pour les cadres atteints par le VIH/sida: la Société nationale d'électricité (Sonel), l'Aluminium du Cameroun (Alucam), pour ne citer que celles-là. Ce foisonnement n'empêche pas que la décision officielle du gouvernement de "bloquer" les prix des médicaments, pour les quelques patients ayant les moyens de se les procurer, soit contrariée de manière récurrente par des ruptures de stocks. Ce qui oblige les malades à se référer à certains médecins qui leur vendent les mêmes produits à un tarif supérieur au prix du marché. Tout ceci signifie simplement que c'est un ensemble hétérogène d'acteurs qui fabrique la lutte contre le sida au Cameroun, en marge du CNLS qui finit par assurer une sorte de "service minimum" au sein de ce champ qui l'intègre et le dépasse.

Le discours du Premier ministre camerounais, le 12 septembre 2000 est donc à lire en ayant à l'esprit les éléments qui précèdent. Son allocution s'est achevée sur un appel que l'on peut estimer contre-productif du fait qu'il pourrait stigmatiser les personnes atteintes et rendre caduque leur engagement qui a la réputation d'être essentiel.

"C'est ici le lieu de condamner avec la dernière énergie les comportements criminels d'individus sans scrupules qui, se sachant infectés par le $\mathrm{VIH} /$ sida, distribuent allègrement la maladie, c'est-à-dire la mort, à de jeunes personnes, victimes innocentes de comportements irresponsables (...). Dans cette perspective, n'est-il pas temps qu'une réflexion éthique soit engagée par la communauté médicale dans Je sens d'envisager la levée du secret médical dans tous les cas où une personne se sachant infectée par le virus du VIH/sida, procéderait volontairement à la contamination d'autres personnes ?" 7.

57 A première vue, on peut souligner que le chef du gouvernement reprend une représentation populaire de la maladie, bâtie sur le mode de la persécution. A ce titre, il suggère les voies de la "sanction", voire de la répression, contraires aux vœux des acteurs de la santé publique et de la lutte contre le sida, dont certains dénoncent les nombreuses atteintes au principe du secret médical. Pourtant, une analyse plus approfondie des quinze ans de lutte contre le sida au Cameroun, impose de relativiser 
l'impact des propos du Premier ministre. Il a rempli une formalité politique, dans un domaine où la "déraison" sanitaire qu'il met en lumière est liée à la "raison politique" qui le fait apparaître ponctuellement dans ce domaine, à travers des circonstances diplomatiques précises. Il agit dans un champ dans lequel il sait que les hommes politiques camerounais ne veulent assurer que le rôle de "guest stars". Son discours semble donc davantage le signe d'un manque avéré de volonté politique - qu'il partage avec beaucoup d'autres responsables politiques en Afrique (Gruénais et al., 1999) - que d'un besoin politique d'infléchir une tendance épidémiologique.

En d'autres termes, le Premier ministre s'adressait davantage à la Banque mondiale qu'à l'ONUSIDA. Les tactiques de gestion des enjeux internationaux occupent les responsables politiques; la question du sida concentre l'énergie des responsables administratifs et sanitaires, locaux et étrangers. De temps à autres, ils se croisent et construisent ensemble un objet qui attend toujours de devenir un objet de débats politiques. Ce qui représente la condition sine qua non pour enrichir la mobilisation (Dozon, 1999) et mettre au centre de la lutte les questions d'éthique et de droits des personnes sans lesquelles les dynamiques épidémiologiques réelles et les réponses sanitaires (donc politiques) demeurent disjointes.

Loin de l'anecdote, cette contradiction apparente entre les "experts" et le "politique" résume de manière symptomatique la situation de l'action contre le sida au Cameroun. Il s'agit d'une politique publique tout à fait réelle effectuée en l'absence de volonté politique. D'où l'expression de "modèle dissonant de politique publique". Tout en sachant que toute politique publique est mue par un ensemble de tractations et d'acteurs qui l'oriente vers des cimes ou des planchers toujours différents des projets initiaux. Le Cameroun ne fait pas exception à la règle.

\section{BIBLIOGRAPHIE}

Bourgeois A., Eboko F, 1997 Situations cliniques et négociations sociales face au VIH/sida au Cameroun, Synthèse critique des "Sessions de formation pour la prise en charge thérapeutique des personnes victimes du VIH/SIDA au Cameroun" des 11 et 12 décembre 1997, Yaoundé, polycop.

Delaunay K., 1999, "Le Programme national de lutte contre le sida au Sénégal. Entre prévention et normalisation sociale", in M.-E. Gruénais (ed.), Organiser la lutte contre le sida en Afrique. Une étude comparative Etat/société civile, Paris, ANRS-IRD ; 101-111.

Dozon J.P., 1999, "Une nécessaire politisation du sida en Afrique", in M.-E. Gruénais (éd.), Organiser la lutte contre le sida en Afrique. Une étude comparative Etat/société civile, Paris, ANRS-IRD, : 51-55.

Eboko F., 2000, "Risque-sida, pouvoirs et sexualité. La puissance de l'Etat en question au Cameroun", in G. Courade (éd.), Le désarroi camerounais. L'épreuve de l'économie-monde, Paris, Karthala : 235-262.

- 1999a, "Logiques et contradictions internationales dans le champ du sida au Cameroun", Autrepart, 12 : 123-140. 
- 1999b, "Les élites politiques au Cameroun : le renouvellement sans renouveau ?", In J.-P. Daloz (éd.)., Le (non-) renouvellement des élites en Afrique subsaharienne, Bordeaux, CEAN : 99-133.

- 1996, "L'État camerounais et les cadets sociaux face à la pandémie du sida", Politique africaine, $64: 135-145$.

Gruenais M.E. (éd.), 1999, Organiser la lutte contre le sida en Afrique. Une étude comparative Etat/ société civile, Paris, ANRS-IRD.

Gruenais M.E., Delaunay K., Eboko F., Gauvrit E., 1999, "Le sida en Afrique : un objet politique ?", Bulletin de l'APAD, Anthropologie de la Santé, 17 :19-36.

Kerouedan D., Eboko F., 1999, Politiques publiques du sida en Afrique, Bordeaux, CEAN, coll. Travaux et Documents.

Ministère de la Santé Publique, Plan cadre de lutte contre le sida au Cameroun. Période 1999-2000, Yaoundé, avril 1999.

Ministère de la Santé Publique, Plan National de Développement Sanitaire du Cameroun 1999-2008, Yaoundé, mai 1998.

Ministère de la Santé Publique, Plan d'action du Ministère de la Santé Publique 1999/2003, Yaoundé, 1998.

Salla Ntounga R., 1996, "Processus du deuxième plan à moyen terme de lutte contre le sida (1994-1998) en République du Cameroun", Bulletin de liaison de l'OCEAC, 26 (4) : 163.

Sam-abbenyi A. (éd.), 1997, HIV/AIDS Surveillance Report 1996, Epidemiology Section, National Aids Control Program Cameroon, Ministry of Public Health.

\section{NOTES}

1.Démis de ses fonctions en 2000.

2.Entretien : Yaoundé, septembre 2000.

3.Jean-François Médard a repris et analysé toutes les formes de décentralisation, à la lumière de l'exemple camerounais. Voir sa contribution dans le présent volume.

4.Entretien réalisé en juin 2000 à Yaoundé au siège de "ONUSIDA.

5.Source : entretien avec M. Festus Wara, épidémiologiste, responsable du FAS Foundation à Bamenda le 14 mai 1999.

6.UNESCO, Actions du Bureau UNESCO de Yaoundé dans le partenariat contre le sida au Cameroun, Répertoire, Yaoundé, 2001,18 p.

7.Pour l'intégralité du discours, voir Cameroon Tribune, $\mathrm{n}^{\circ}$ 7184/3473 du 13 septembre 2000 , p. 4. 


\section{AUTEUR}

FRED EBOKO

Institut d'Études Politiques, Domaine universitaire BP 101 - 33405 Talence ;

fred.eboko@wanadoo.fr 